\title{
Sentinel lymph node biopsy in thyroid cancer - literature review
}

\author{
Nicolae BACALBASA ${ }^{1,2,3}$, Irina BALESCU ${ }^{4}$, Mihaela VILCU ${ }^{2,3}$, Iulian BREZEAN ${ }^{2,3}$ \\ ${ }^{1 " I o n ~ C a n t a c u z i n o " ~ C l i n i c a l ~ H o s p i t a l, ~ B u c h a r e s t, ~ R o m a n i a ~}$ \\ 2"Carol Davila" University of Medicine and Pharmacy, Bucharest, Romania \\ ${ }^{3}$ Center of Excellence in Translational Institute, Fundeni Clinical Institute, \\ Bucharest, Romania \\ ${ }^{4}$ Ponderas Academic Hospital, Bucharest, Romania
}

\begin{abstract}
Thyroid cancer has been usually treated by total or near total thyroidectomy in association with cervical lymph node dissection and followed by adjuvant oncological and endocrinological treatment. In order to maximize the effect of the surgical approach and to minimize the perioperative rates of complications, certain authors proposed the introduction of the sentinel node detection; however, the method has certain particularities due to the complex lymphatic anatomy of the cervical region. This is a literature review of the more significant studies conducted so far on this theme.
\end{abstract}

Keywords: thyroid cancer, cervical lymph node dissection, sentinel node biopsy

\section{INTRODUCTION}

Papillary thyroid carcinoma represents the most commonly encountered endocrine tumor with a high propensity of spread via lymphatic route, leading to the apparition of metastatic disease, especially at the level of the central compartment of the neck $(1,2)$.

Currently, the standard therapeutic approach in thyroid carcinoma consists of total or near total thyroidectomy in association with locoregional lymph node dissection, iodine 131 treatment, TSH suppression and thyroid hormonal replacement. The improvement of the imagistic techniques conducted to a better identification of the suspected lymph nodes and offered the possibility for the surgeon to perform a less extended surgical procedure, diminishing in this way the perioperative complication rates with similar results in regard to the long term outcomes (3). However, currently there is a permanent debate in regard with the indication of lymph node dissection; while certain authors consider that prophylactic lymph node dissection should be performed in all cases, other authors consider that lymph node dissection should be reserved only for patients presenting imagistic or intraoperative signs of lymph node metastases $(4,5)$.

Once the concept of sentinel node biopsy has been widely implemented in breast cancer or malignant melanoma, its' utility has been widely analyzed in other pathological entities with encouraging results $(6,7)$. In the last decade the method was also proposed as part of the therapeutic approach in thyroid cancer. The method was initially considered skeptical due to the complex lymphatic drainage from the level of the cervical region; it is well known the fact that the lymphatic stations are located in the 
close proximity of the vessels and nerves, which, at the level of the cervical region, present a high number of anatomical variants $(3,8)$.

\section{Lymphatic drainage of the thyroid gland}

Traditionally, it has been described the presence of a superior lymphatic drainage from the isthmus and the superior part of the thyroid lobes which terminate in the subdigastric nodes, part of the internal jugular chain, a median and inferior lymphatic station draining in the pretracheal nodes and a lateral one draining into the superior part of the jugular chain and to the inferior part of the internal jugular chain (3).

\section{The influence of lymphatic metastases in thyroid cancer patients}

It has been demonstrated that the presence of lymph node metastases in thyroid cancer patients is associated with a significantly worsened prognostic; moreover, the presence of extracapsular invasion represents another significant prognostic factor in regard to the risk of developing distant metastases $(9,10)$. In consequence, an appropriate knowledge of the status of the lymph nodes is mandatory in order to orientate the adjuvant therapy in such cases and the long term prognostic.

\section{Techniques of sentinel node detection in thyroid cancer patients}

Sentinel node detection in thyroid cancer can be performed after injection of colored agents such as isosulfan blue or after injection of radiocolloid tracers, such as Technetium 99. If colored dye is chosen for injection, multiple protocols have been proposed. The dye can be injected directly into the suspected nodule into a single site or at multiple levels (at 3 o'clock, 6 o'clock, 9 o'clock and 12 o'clock around the tumoral nodule). However, most authors consider that the injection should be performed before dissecting the gland in order not to interrupt the lymphatic channels; once injected, the dye will concentrate at the level of the sentinel nodes in few seconds up to 2 minutes; an important aspect related to this technique is the one regarding the possible coloration of the parathyroid gland which might predispose to confusion between these structures and the sentinel nodes. Another problem which might be encountered after using this technique is the one related to the identification of the sentinel nodes at the level of the central compartment, which might be difficult to be exposed within in a few minutes after injection $(3,11-13)$.

In order to prevent the apparition of these complications, other authors suggest the utility of technetium injection at the level of the tumoral nodule one day before surgery. If this technique is chosen to be applied, attention should be carried out in order not to inject the substance in the peritumoral area, due to the high risk of spillage consecutively to the rich vascularization of the thyroid tissue (14). One day later thyroidectomy is performed; after ending the thyroid removal, a gamma probe is used in order to detect the radioactivity in the central and lateral compartments; once all suspected tissues are removed, the remnant radioactivity of the surgical bed should be also verified in order not to omit excision of any radioactive structure. The timing - thyroid removal followed by sentinel node detection should be exactly followed; if sentinel nodes are searched before removing the gland, interference between the radioactivity of the gland and of the nodes could affect the accuracy of the technique $(3,15)$.

In order to maximize the process of sentinel node detection, certain authors reported the use of a dual method (radiocolloid and dye injection) with promising results (16).

Another method which has been proposed with promising results was the one which uses carbon nanoparticles. This method consists of injecting carbon particles with a mean diameter of $150 \mathrm{~nm}$ in the close proximity of the tumor, which are rapidly engulfed by the macrophages and accumulate in the sentinel nodes, staining them in black (17).

The method was successfully reported by the Chinese surgeons on a group of 100 patients; further on, they compared their results to the ones reported in a similar group of 100 patients in whom methylene blue is injected. The authors reported a significantly improved rate of detection for the sentinel nodes among cases in which carbon nanoparticles had been injected; moreover, the sensibility, specificity accuracy rate and false negative rate were improved among patients submitted to carbon injection, while the rate of micrometastatic retrieved nodes was also significantly higher when compared to the methylene blue group $(61.1 \%$ versus $47.1 \%, p=0.034$ ); as for the method of detection, in all cases injection of the agent was performed just after exposure of the thyroid gland and of the ipsilateral jugular vein into the 
parenchyma surrounding the nodule; moreover the authors underlined the fact that in the methylene blue injection group there were four cases in which the resected tissue stained in blue was in fact the parathyroid gland, while in carbon nanoparticles injection group this error did not occur (17).

A more recent study conducted by Fustegueras et al. (18) on 20 patients with papillary thyroid carcinoma demonstrated the utility of magnetic guided sentinel node biopsy using superparamagnetic iron oxide, a tracer which has been used in the last two decades as an intravenous contrast agent for magnetic resonance imaging studies (19). Using this method, the Spanish surgeons detected a median number of two sentinel nodes/patient, while the ratio of metastatic nodes was of $31 \%$; among the retrieved non-sentinel nodes, the ratio of positive nodes was $10.2 \%$, all cases of positive non-sentinel nodes being found among patients with positive sentinel nodes. Therefore, the authors demonstrated that the sensitivity of the method was $100 \%$, the specificity was $81.8 \%$, while the accuracy was $87.5 \%$, underlining in this way the effectiveness of the method (18).

Another interesting study coming also from Spain which demonstrated the utility of sentinel node biopsy in thyroid carcinoma patients was conducted by Gonzalez and was published in 2017 (20). The study included 43 patients submitted to thyroidectomy and sentinel node dissection; after surgery, the authors demonstrated that 20 of the 30 patients diagnosed with presumed early stage disease (T1 and T2) were finally identified as having lymph node metastases, whereas 12 of the 13 cases diagnosed in advanced stages of the disease (T3 and T4) were also diagnosed with lymphatic tumoral involvement. The utility of the method was further demonstrated by the fact that finally there was no false positive result, while in four cases the only positive node was the sentinel one, the rest of the excised nodes as part of the lymph node dissection proving to be negative (20).

However, the method is not widely accepted by all the studies; for example, in a study conducted by the Korean surgeons at Samsung Medical Center, the authors included 283 patients with thyroid papillar carcinoma submitted to surgery (21). The patients were randomized for sentinel node dissection and central node dissection in association with total or near total thyroidectomy; finally there were 142 cases submitted to lymph node dissection and 141 cases submitted to sentinel node biopsy; in cases in which sentinel node biopsy was performed, radiocolloid technetium was chosen as tracer and was injected one day before surgery. The authors reported no significant difference among the two groups in regard of the tumor location, age, sex, number of nodules, tumoral extent, and association of thyroiditis or resection margin status. However, although sentinel node biopsy was able to remove the occult disease, this fact did not influence the recurrence rate after a mean follow-up of 39 months; therefore, the authors concluded that further studies are still needed in order to establish the clinical utility of the method (21).

\section{CONCLUSIONS}

Sentinel node detection seems to be a reliable and safe method in thyroid cancer patients which might provide a better identification of the cases at risk to develop further metastases and, in consequence, to have a poorer outcome. As for the type of injected agent, it seems that better results are obtained after technetium or carbon nanoparticles injection. Moreover, the use of the superparamagnetic iron oxide seems to be associated with promising results.

Conflict of interest: none declared Financial support: none declared

\section{REFERENCES}

1. Hundahl SA, Fleming ID, Fremgen AM et al. A National Cancer Data Base report on 53,856 cases of thyroid carcinoma treated in the U.S., 1985-1995 [see commetns]. Cancer 1998; 83: 2638-48.

2. Zafon $\mathrm{C}$, Puig-Domingo $\mathrm{M}$, Biarnes $\mathrm{J}$ et al. A descriptive study of the characteristics of differentiated thyroid cancer in Catalonia during the period 1998-2012. The CECaT registry. Endocrinol. Nutr. 2015; 62: 264-9.
3. Rubello D, Pelizzo MR, Al Nahhas A et al. The role of sentinel lymph node biopsy in patients with differentiated thyroid carcinoma. Eur. J. Surg. Oncol. 2006; 32: 917-21.

4. Zhu W, Zhong M, Ai Z. Systematic evaluation of prophylactic neck dissection for the treatment of papillary thyroid carcinoma. Jpn. J. Clin. Oncol. 2013; 43: 883-8.
5. Lang BH, $\mathrm{Ng} \mathrm{SH}$, Lau LL et al. A systematic review and meta-analysis of prophylactic central neck dissection on short-term locoregional recurrence in papillary thyroid carcinoma after total thyroidectomy. Thyroid 2013; 23: 1087-98.

6. Haigh PI, Giuliano AE. Sentinel lymph node dissection for thyroid malignancy. Recent Results Cancer Res. 2000; 157: 201-5. 
7. Mariani G, Gipponi M, Moresco L et al. Radioguided sentinel lymph node biopsy in malignant cutaneous melanoma. J. Nucl. Med. 2002; $43: 811-27$.

8. Gregoire V, Coche E, Cosnard G et al. Selection and delineation of lymph node target volumes in head and neck conformal radiotherapy. Proposal for standardizing terminology and procedure based on the surgical experience. Radiother. Oncol. 2000; 56: 135-50.

9. Yamashita $\mathrm{H}$, Noguchi $\mathrm{S}$, Murakami $\mathrm{N}$ et al. Extracapsular invasion of lymph node metastasis is an indicator of distant metastasis and poor prognosis in patients with thyroid papillary carcinoma. Cancer 1997; 80: 2268-72.

10. Ohshima A, Yamashita H, Noguchi S et al. Indications for bilateral modified radical neck dissection in patients with papillary carcinoma of the thyroid. Arch. Surg. 2000; 135: 1194-8.

11. Kelemen PR, Van Herle AJ, Giuliano AE. Sentinel lymphadenectomy in thyroid malignant neoplasms. Arch. Surg. 1998; 133: 288-92.
12. Fukui Y, Yamakawa T, Taniki T et al. Sentinel lymph node biopsy in patients with papillary thyroid carcinoma. Cancer 2001; 92: 2868-74.

13. Stoeckli SJ, Pfaltz M, Steinert $\mathrm{H}$ et al. Sentinel lymph node biopsy in thyroid tumors: a pilot study. Eur.Arch. Otorhinolaryngol. 2003; 260: 364-8.

14. Takami H, Sasaki K, Ikeda Y et al. Sentinel lymph node biopsy in patients with thyroid carcinoma. Biomed.Pharmacother. 2002; 56 Suppl 1: 83s-7s.

15. Wiseman SM, Hicks WL, Jr., Chu QD et al. Sentinel lymph node biopsy in staging of differentiated thyroid cancer: a critical review. Surg. Oncol. 2002; 11: 137-42.

16. Catarci M, Zaraca F, Angeloni R et al. Preoperative lymphoscintigraphy and sentinel lymph node biopsy in papillary thyroid cancer. A pilot study. J.Surg. Oncol. 2001; 77: 21-4.

17. Hao RT, Chen J, Zhao LH et al. Sentinel lymph node biopsy using carbon nanoparticles for Chinese patients with papillary thyroid microcarcinoma. Eur.J.Surg.Oncol. 2012; 38: 718-24.
18. Baena Fustegueras JA, González FH, Calderó SG et al. Magnetic detection of sentinel lymph node in papillary thyroid carcinoma: The MAGIC-PAT study results, European Journal of Surgical Oncology, in press, https://doi.org/10.1016/j. ejso.2019.03.017

19. Weissleder R, Elizondo G, Wittenberg J et al. Ultrasmall superparamagnetic iron oxide: an intravenous contrast agent for assessing lymph nodes with MR imaging. Radiology 1990; 175: 494-8.

20. Gonzalez O, Zafon C, Caubet E et al. Selective sentinel lymph node biopsy in papillary thyroid carcinoma in patients with no preoperative evidence of lymph node metastasis. Endocrinol. Diabetes Nutr. 2017; 64: 451-5.

21. Lee SK, Lee JH, Bae SY et al. Lateral neck sentinel lymph node biopsy in papillary thyroid carcinoma, is it really necessary? A randomized, controlled study. Surgery 2015; 157: 518-25. 